\title{
Influence of Capillary Number on Pressure Profile Evolution in Microfluidic T-Junction
}

\author{
Piyush Kumar', Manabendra Pathak ${ }^{1}$ \\ ${ }^{1}$ Indian Institute of Technology Patna \\ Bihta, Patna, India - 801103 \\ piyush.pme16@iitp.ac.in; mpathak@iitp.ac.in
}

\begin{abstract}
A three dimensional, numerical model has been developed to predict variation of pressure during droplet formation in microfluidic T-junction. Investigations have been carried out to examine the influence of capillary number on pressure variation during the entire cycle of droplet generation. With increase in Capillary number, width of dispersed phase in main channel decreases and the hydrodynamic resistance to flow of continuous phase fluid decreases, resulting in a smaller pressure drop along the microchannel. Pressure difference across the thread of dispersed phase is calculated during the droplet formation for different values of Capillary number. The pressure difference across the thread of dispersed phase decreases with increase in Capillary number. The frequency of upstream pressure fluctuations coincides with the formation of the droplet, and the amplitude and pitch of the pressure fluctuations varies with the Capillary number.
\end{abstract}

Keywords: Microfluidic Droplet generation; T-junction; two phase Level SET method (LSM).

\section{Introduction}

Droplet-based microfluidics offers a wide range of applications in the field of chemical and biological analysis, protein crystallization, encapsulation of cells, synthesis of advanced materials and digital polymerase chain reaction (PCR) systems. Three prominent techniques are adopted for generating uniform droplets i.e. droplet generation in co-flowing streams, droplet generation by stretching or elongation shearing and droplet formation in crossflowing streams. Cross flowing stream of two immiscible fluids is one of the most common droplet generator designs due to its simplicity in geometric configuration and superior control over droplet size. In this design, droplets are generated from dispersed phase which perpendicularly intercepted by continuous phase. For modelling two phase flows, several multiphase approaches which include volume-offluid (VOF) [1, 2], Lattice-Boltzmann methods (LBM) [3], and phase field [4] have been reported in the literature.

During droplet formation, pressure fluctuations inside microchannel can occur due to flow-rate instability arising from the pump and the advancement of dispersed phase fluids in the main channel during droplet generation. Garstecki et al. [5] studied the evolution of continuous phase pressure and assumed that dispersed phase pressure remains constant during droplet formation. Menech [4] performed a parametric study of pressure profile in relation to variations in interfacial tension. However, he did not include the dependency of contact angle and flow rate of continuous phase. Yan et al. [6] numerically investigated the junction point pressure variation for the entire regime of droplet formation. Later on, Bashir et al. [7] performed the numerical simulation to predict the dependency of flow rate of continuous phase, wettability and interfacial tension on pressure profile. Zeng et al. [8] theoretically calculated the magnitude of pressure fluctuations for the different geometry and compared its results with the experimental data.

It has been observed that role of Capillary number $\left(\mathrm{Ca}=\mu_{c} Q_{c} / \sigma w h\right)$ on the pressure variation has not been fully investigated. So, evolution of pressure profile near the junction needs to be further investigated. The objective of the present work is to develop a numerical model for investigation of pressure profile evolution in microfluidic T-junction with varying $\mathrm{Ca}$. The effect of $\mathrm{Ca}$ on pressure profiles has been investigated numerically with help of laminar two phase Level-SET method (LSM).

\section{Model Description}




\subsection{Geometry and Boundary Conditions}

The schematic of T-junction geometry used in the simulation is shown in Figure 1. The geometry has two inlets with cross-section of $100 \times 100 \mu \mathrm{m}^{2}$. The junction point, where the both phases interact is kept at a distance of $200 \mu \mathrm{m}$ from the starting point of the continuous phase. Simulations have been performed with half geometry due to symmetry. At both inlets, laminar inflow volumetric flow rates have been used. At the outlet, atmospheric pressure is defined. The wetted wall boundary condition has been applied to all solid boundaries with the contact angle equal to $135^{\circ}$.

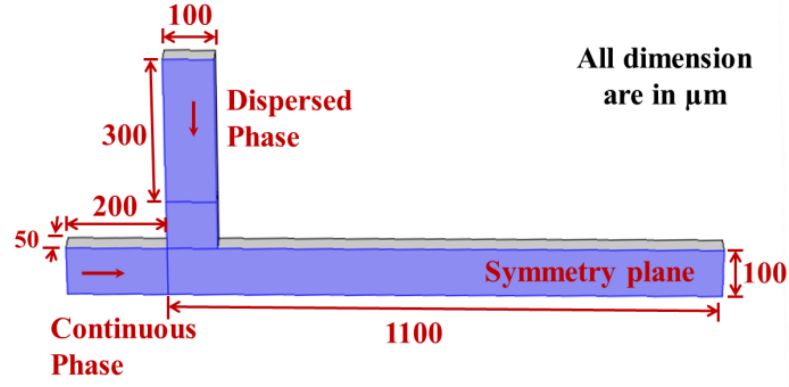

Fig. 1: Schematic of T-junction.

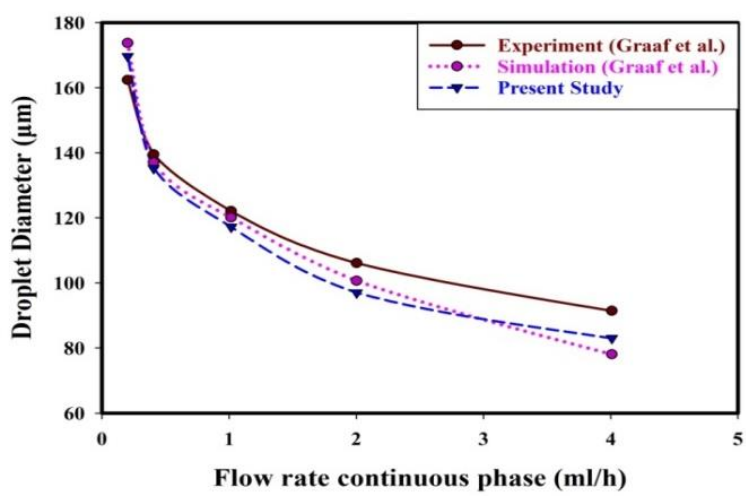

Fig. 2: Droplet diameter as a function of the flow rate of the continuous phase at $\mathrm{Q}_{\mathrm{d}}=0.2 \mathrm{ml} / \mathrm{h}, \lambda=3.44$ and $\theta=135^{\circ}$.

\section{2. Laminar two-phase flow Level-SET method}

3-D numerical simulations of microfluidic droplet formation in T-shaped geometry have been done by using laminar two phase LSM. Governing equations of the problem comprise of continuity, Navier-Stokes equation and transport equation of level set function.

$$
\begin{gathered}
\nabla \cdot u=0 \\
\rho \frac{\partial u}{\partial t}+\rho(u \cdot \nabla) u=\nabla\left[-p I+\mu\left(\nabla u+(\nabla u)^{T}\right)\right]+F_{s t} \\
\frac{\partial \phi}{\partial t}+\nabla \cdot(u \phi)=\gamma \nabla \cdot\left[\varepsilon \nabla \phi-\phi(1-\phi) \frac{\nabla \phi}{|\nabla \phi|}\right.
\end{gathered}
$$

Where $\mu . \rho, \varepsilon, \gamma, \sigma, p$ and I denote the dynamic viscosity, density, the artificial thickness of the interface, reinitialization parameter, interfacial tension between two immiscible fluids, pressure and identity matrix respectively. Surface tension force at the interface between two immiscible fluids are determined by:

$$
F_{s t}=\nabla \cdot\left(\sigma\left(I-\left(n n^{T}\right)\right) \delta\right)
$$

Where $\mathrm{n}$ and $\delta$ denote unit normal vector, Dirac delta function respectively. Dirac delta function is obtained by

$$
\delta=6 \mid \phi(1-\phi) \| \nabla \phi
$$

Density and dynamic viscosity of combined flow are expressed as a function of Level-SET function as shown below:

$$
\begin{aligned}
& \rho=\rho_{1}+\left(\rho_{2}-\rho_{1}\right) \phi \\
& \mu=\mu_{1}+\left(\mu_{2}-\mu_{1}\right) \phi
\end{aligned}
$$


Where subscript 1 and 2 denotes the continuous phase and dispersed phase respectively. Two-phase LSM model has been used to solve all these equations in COMSOL Multiphysics.

\section{3. Grid convergence and model validation}

Prior to parametric study, mesh convergence analysis was carried out to check the dependency of numerical results on mesh size. A 3D quadrilateral mapped mesh has been used in which the number, size and distribution of the element can be controlled using size and distribution sub nodes. To examine the effect of mesh size, number of domain element was changed and optimum mess size was selected taking in view of convergence time and numerical results.

After grid convergence analysis, numerical model was validated with the experimental and numerical results of Graaf et al. [3] as shown in Figure 2. The density of continuous and dispersed phase fluids are set as $1.00 \times 10^{3} \mathrm{~kg} / \mathrm{m}^{3} \mathrm{and} 1.02 \times 10^{3} \mathrm{~kg} / \mathrm{m}^{3}$, with the dynamic viscosities of $1.95 \times 10^{-3} \mathrm{~Pa} . \mathrm{s}$ and $6.71 \times 10^{-3} \mathrm{~Pa}$.s respectively. The interfacial tension $(\sigma)$ between two immiscible fluids are $0.005 \mathrm{~N} / \mathrm{m}$.

\section{Results and discussion}

A series of simulation, with different Capillary number has been performed to investigate the evolution of pressure across the thread of dispersed phase in microfluidic T-junction. In simulations, $\mathrm{Ca}$ is varied by changing the interfacial tension $(\sigma)$ between two immiscible fluids. During numerical simulation flow rate ratio $(\varphi=0.2)$, wettability $\left(\theta=135^{\circ}\right)$ and viscosity ratio $\left(\lambda=\mu_{\mathrm{d}} / \mu_{\mathrm{c}}=12\right)$ is kept constant. The droplets generation in T-junction is dominated by pressure drop along the thread and shear stress exerted by continuous phase on thread of dispersed phase, while interfacial tension resists the deformation of emerging thread. With the increase in Ca number or decrease in $\sigma$, the width of emerging thread in continuous phase channel decreases as shown in pressure contour of Figure 4. Figure 3 shows the location of droplet in continuous phase at the same instant of time $\mathrm{T}=\mathrm{t} / \mathrm{t}_{\max }=0.68$ for different Ca. Location of droplet in continuous phase show the droplet formation time decreases with increase in $\mathrm{Ca}$. This can be concluded as the frequency of droplet formation increase with increase in Ca.

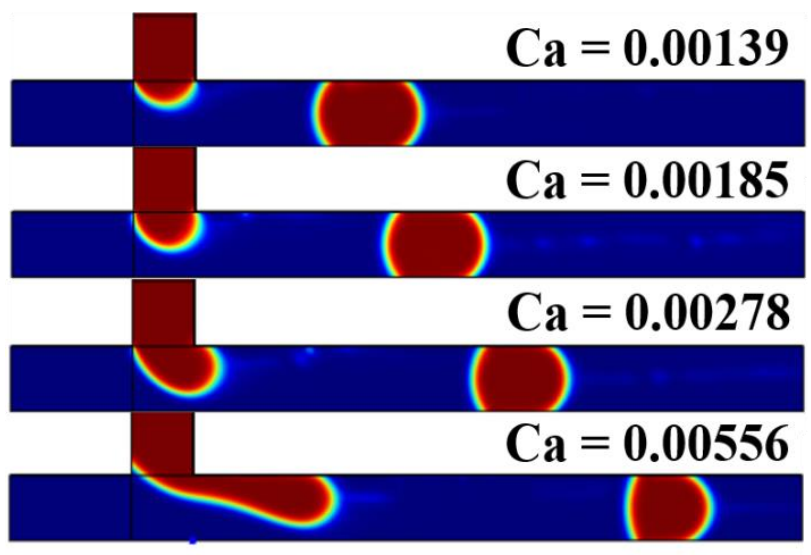

Fig. 3: Distributions of dispersed phase at $\mathrm{T}=0.68$ for different $\mathrm{Ca}$ at $\theta=135^{\circ}, \lambda=12$ and $\mathrm{Q}=0.2$.

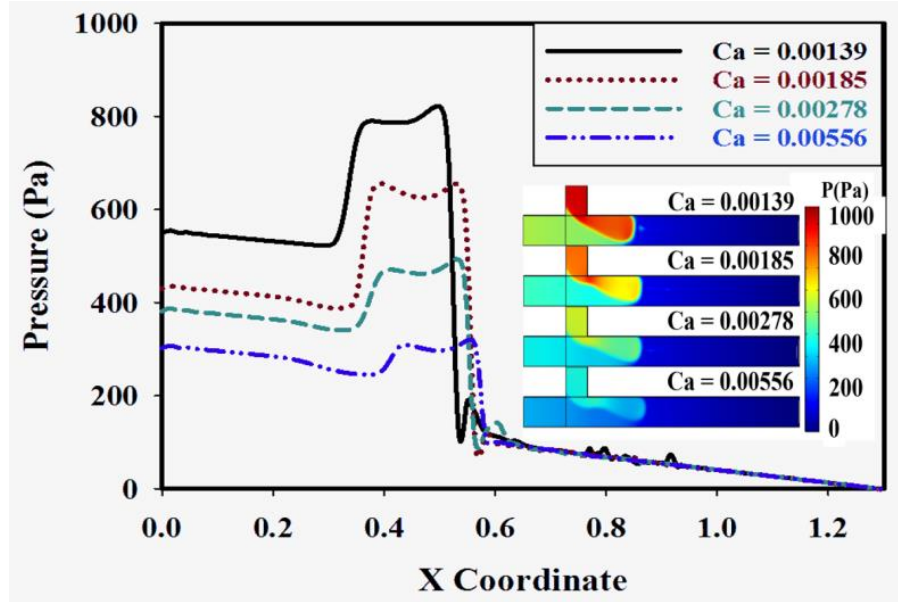

Fig. 4: Effect of $\mathrm{Ca}$ on pressure variation along $\mathrm{x}$ direction at the central axis of main channel on symmetry plane.

Figure 4 shows the pressure variation along $\mathrm{x}$-direction at the central axis of main channel on symmetry plane before the break-up of droplet from dispersed phase fluid. The pressure decreases along the x-direction for all the value of Ca. The pressure fluctuations are due to change in surface tension between two immiscible fluids. The pressure fluctuation appears near the junction of side and main channel where dispersed phase penetrates into the main channel. It can also be observed that the pressure gradient alone the x-direction decreases with increase in Ca. Pressure gradient across the thread of dispersed 
phase fluid decreases with the increase in Ca. As Ca increases, width of dispersed phase in main channel decrease and the hydrodynamic resistance to the flow of continuous phase fluid decreases, resulting in a smaller pressure drop along the microchannel.

The pressure difference (upstream pressure $\mathrm{P}_{\mathrm{A}}$ - downstream pressure $\mathrm{P}_{\mathrm{B}}$ ) across the thread is one of the important force for the detachment of the droplet. Figures 5 show the time evolution of pressure difference across the thread of dispersed phase in the microfluidic T-junctions for different value of $\mathrm{Ca}$. As the thread of dispersed phase block the cross-section of the main channel, the pressure difference across the thread increases with time. Due to the fixed outlet pressure, the pressure difference across the thread of dispersed phase decrease with increase in $\mathrm{Ca}$.

Figure 6 shows the variation of upstream pressure at point $\mathrm{A}$ with time for different value of $\mathrm{Ca}$. With increase in $\mathrm{Ca}$ or decrease in $\sigma$, pressure decreases in the main channel. For $\mathrm{Ca}=0.00556$, the amplitude of pressure fluctuation is less in comparison of $\mathrm{Ca}=0.00139$. However, frequency of pressure fluctuation is more in case of high $\mathrm{Ca}$ as compared to low $\mathrm{Ca}$. It can also be seen that at $\mathrm{Ca}=0.0139$ (higher surface tension), the pressure drastically falls after the droplet breakup, which may be due to strong pull back (lag stage) of dispersed phase after droplet formation. The peak value of pressure decreases with increase in $\mathrm{Ca}$, which is due to decease in the width of dispersed phase in main channel.

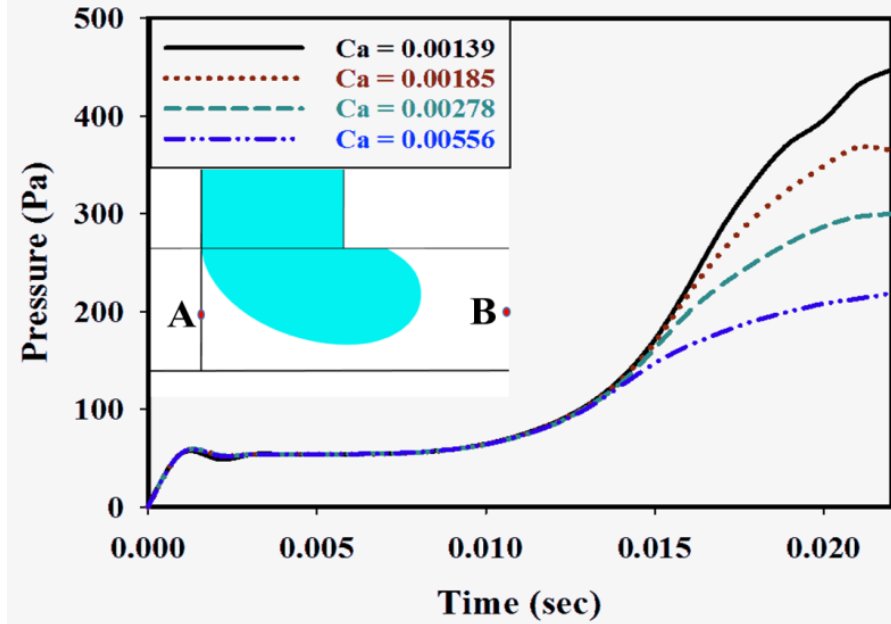

Fig. 5: The variation of pressure difference $\left(\mathrm{P}_{\mathrm{A}}-\mathrm{P}_{\mathrm{B}}\right)$ across the thread with time during the droplet formation at $\theta=135^{\circ}, \lambda=$ 12 and $\mathrm{Q}=0.2$

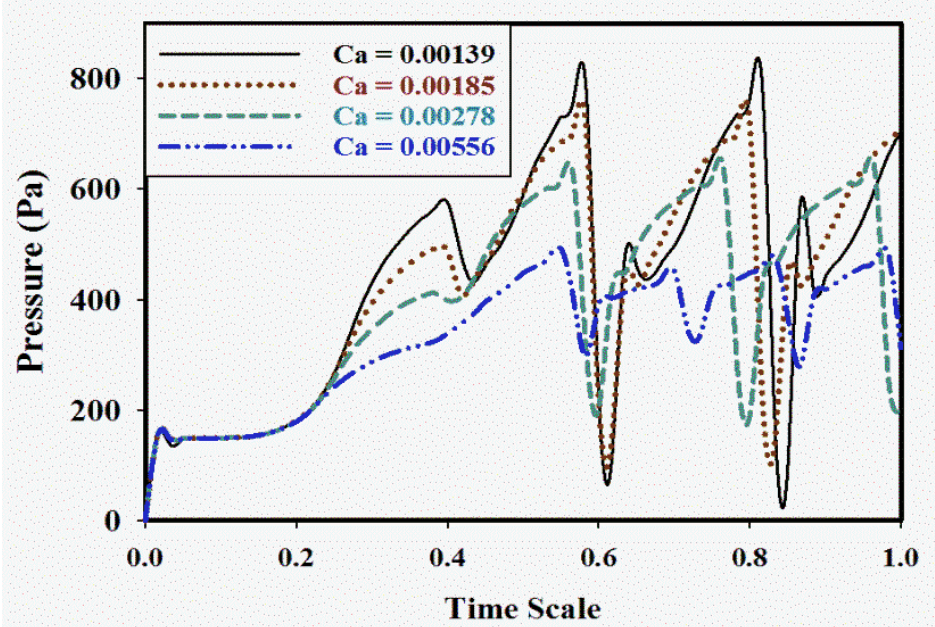

Fig. 6: variation of upstream pressure at point A for different value of $\mathrm{Ca}$ at $\theta=135^{\circ}, \lambda=12$ and $\mathrm{Q}=0.2$.

\section{Conclusion}

Conservative two phase Level-SET method has been successfully used for the numerical investigation of droplet formation in T-junction. Detailed investigation of pressure profile near the junction has been presented for different value of $\mathrm{Ca}$. Pressure contour reveals that the pressure in dispersed phase is always higher than the upstream and downstream pressure of continuous phase. Upstream pressure is higher than downstream pressure along the droplet, which helps the dispersed phase to flow in the downstream direction in main channel. As Ca increases, width of dispersed phase in main channel decrease and the hydrodynamic resistance to flow of continuous phase fluid decreases, resulting in a smaller pressure drop along the microchannel. The pressure difference (upstream pressure $\mathrm{P}_{\mathrm{A}}$ - downstream pressure $\mathrm{P}_{\mathrm{B}}$ ) across the thread of dispersed phase decrease with increase in $\mathrm{Ca}$. It has also been observed that as Ca increases, the frequency of droplet formation increases but the amplitude and pitch of the upstream pressure decreases during the droplet formation. Maximum value of upstream pressure decreases with increase in $\mathrm{Ca}$.

\section{References}


[1] Mehdi Nekouei and Siva A. Vanapalli, "Volume-of-fluid simulations in microfluidic T-junction devices: Influence of viscosity ratio on droplet size," Phys. FLUIDS, vol. 29, no. 3, 2017.

[2] M. Pathak, "Numerical simulation of membrane emulsification: Effect of flow properties in the transition from dripping to jetting," J. Memb. Sci., vol. 382, no. 1-2, pp. 166-176, 2011.

[3] S. Van Der Graaf, T. Nisisako, C. G. P. H. Schroën, R. G. M. Van Der Sman, and R. M. Boom, "Lattice Boltzmann simulations of droplet formation in a T-shaped microchannel," Langmuir, vol. 22, no. 9, pp. 4144-4152, 2006.

[4] M. De Menech, "Modeling of droplet breakup in a microfluidic T-shaped junction with a phase-field model," Phys. Rev. E - Stat. Nonlinear, Soft Matter Phys., vol. 73, no. 3, pp. 1-9, 2006.

[5] P. Garstecki, M. J. Fuerstman, H. A. Stone, and G. M. Whitesides, "Formation of droplets and bubbles in a microfluidic T-junction - Scaling and mechanism of break-up," Lab Chip, vol. 6, no. 3, pp. 437-446, 2006.

[6] Y. Yan, D. Guo, and S. Z. Wen, "Numerical simulation of junction point pressure during droplet formation in a microfluidic T-junction," Chem. Eng. Sci., 2012.

[7] S. Bashir, J. M. Rees, and W. B. Zimmerman, "Investigation of pressure profile evolution during confined microdroplet formation using a two-phase level set method," Int. J. Multiph. Flow, 2014.

[8] W. Zeng, S. Li, and H. Fu, "Modeling of the pressure fluctuations induced by the process of droplet formation in a Tjunction microdroplet generator," Sensors Actuators, A Phys., vol. 272, pp. 11-17, 2018. 